\title{
Novedades informáticas para la gestión de archivos en el ámbito municipal de la provincia de Zaragoza
}

\author{
The current versions of software for the management of municipal archives in the province of Zaragoza (Spain)
}

\section{Ana Lucía Esteban SÁnchez (1), José Francisco EgeA GiLABerte (2), Guillermo Cano de GuAdALFA- JARA (3), Daniel Sebastián Cetina (4)}

(1) Escuela Universitaria de La Almunia, C/ Mayor, s/n, 50100 La Almunia (Zaragoza), anaeste@unizar.es (2) Diputación Provincial de Zaragoza, Plaza de España, 2, 50071 Zaragoza, jfegea@dpz.es (3) gcano@dpz.es (4) dsebastian@dpz.es

\begin{abstract}
Resumen
Se describen las versiones actuales de tres de los programas informáticos surgidos de la colaboración entre la Diputación Provincial de Zaragoza y la Escuela Universitaria Politécnica de La Almunia, empleados para una mejor gestión de los archivos municipales. Para ello, se ofrece una visión global de las características funcionales más relevantes de dichas herramientas informáticas, una de las cuales está siendo utilizada por numerosos ayuntamientos de la provincia; otra está pensada para su uso únicamente en municipios de unas determinadas características; y la tercera restringida a la Diputación Provincial de Zaragoza. Se muestran gráficamente algunas de las funcionalidades más significativas de las mencionadas herramientas informáticas.
\end{abstract}

Palabras clave: Sistemas de gestión automatizada de archivos. Diputación Provincial de Zaragoza. Escuela Universitaria Politécnica de La Almunia. Normalización. Archivos municipales.

\section{Introducción}

El Departamento de Archivos Municipales, que tiene su origen en 1983, es el órgano de la Diputación Provincial de Zaragoza (en adelante DPZ) encargado de dar asistencia técnica a los ayuntamientos para que éstos dispongan de un servicio de archivo. Actualmente, se ha desarrollado un modelo de gestión compartida de estos archivos entre la institución provincial y los ayuntamientos titulares de los fondos documentales, siendo un modelo flexible para adaptarse a los cambios que se producen en el ámbito de nuestra área de responsabilidad (1).

En 1990, la DPZ comenzó la informatización de los instrumentos de recuperación de los fondos archivísticos con los que trabajaba (2), justificada por las ventajas que ofrece esta tecnología frente a los ficheros tradicionales.

El actual conjunto de herramientas informáticas de que dispone el Departamento de Archivos

\begin{abstract}
We describe the three current versions of software that are arising from the collaboration between the Diputación Provincial de Zaragoza and the Escuela Universitaria Politécnica de La Almunia. They are used for better management of the municipal archives in the province of Zaragoza (Spain). For this purpose, an overview of the most important functional characteristics of these tools is provided, one of which is being used by many municipalities in the province, another is intended for use only in municipalities with certain characteristics, and the third one is restricted to the Diputación Provincial de Zaragoza.
\end{abstract}

Keywords: Records management systems. Diputación Provincial de Zaragoza (Spain). Escuela Universitaria Politécnica de La Almunia (Zaragoza, Spain). Municipal archives. Standardization.

Municipales de la DPZ es el resultado del intenso trabajo realizado durante las dos últimas décadas.

Aun cuando en el presente texto hacemos referencia únicamente a archivos municipales de la provincia de Zaragoza -que son el ámbito natural de actuación del citado Departamento-, hemos de tener en cuenta que algunas de estas herramientas están siendo empleadas también por juzgados municipales, comarcas, notarías, registros de la propiedad y otras instituciones y por ayuntamientos de otras provincias-, lo que muestra su versatilidad. La DPZ distribuye gratuitamente dichas herramientas.

A continuación se describe brevemente dicha evolución, desde la informatización inicial de fichas, hasta la actual gestión normalizada de archivo. Seguidamente se exponen las herramientas informáticas más importantes y en uso actualmente. Todas ellas están relacionadas y, 
en conjunto, abarcan la mayoría de las etapas del ciclo de vida del documento.

\section{Evolución}

En 1999 se firmó un convenio de colaboración entre la DPZ y la Escuela Universitaria Politécnica de La Almunia (en adelante EUPLA), cuyo principal fruto habían sido las sucesivas versiones del programa Veruela, pensado fundamentalmente para mecanizar los inventarios e índices de los fondos que se iban organizando. Se trata, por lo tanto, de una herramienta de gestión del archivo, caracterizada por su sencillez de manejo, para poder ser utilizada en los ayuntamientos por personal sin una especial formación archivística ni informática.

La necesidad de dar respuesta a problemas y circunstancias de diversa índole, que ahora analizaremos, han aconsejado aprovechar este marco de colaboración entre DPZ y EUPLA para, por un lado, realizar una nueva versión de Veruela, y por otro, para crear otros dos programas, uno, llamado Moncayo, para la gestión del archivo de oficina; y el otro, Sástago, para la gestión normalizada de la descripción documental, utilizando los estándares de intercambio de datos establecidos por las normas del Consejo Internacional de Archivos (en adelante CIA). La presentación de estas tres novedades es el objetivo del presente trabajo.

Las tres son herramientas informáticas relacionadas y complementarias. Todas ellas están diseñadas para facilitar diversas tareas sobre la gestión de los fondos. Son herramientas para trabajar en red, diseñadas como aplicaciones informáticas de base de datos centralizada.

\section{Estado actual}

La figura 1 muestra un esquema con las herramientas, en sus diferentes situaciones y modalidades de trabajo. Por las propias características de los programas, Veruela está implantado en numerosos municipios; Moncayo sólo en los de determinado tamaño y circunstancias; y Sástago se utiliza únicamente en la DPZ.

Periódicamente los ayuntamientos envían sus bases de datos (BBDD) de Veruela a la DPZ, para su conservación. Si es necesario, los archiveros de la Diputación modifican esa base de datos para homogeneizar criterios de clasificación, descripción o indización, en cuyo caso la devuelven actualizada al municipio. En la DPZ también se ponen las bases de datos a disposición de los usuarios para su consulta, dentro de los términos que permite la legislación vigente. De este modo, la DPZ asume (de forma com- partida con los distintos ayuntamientos) la tarea de conservación de las copias de seguridad y de hacerlas accesibles a los ciudadanos.

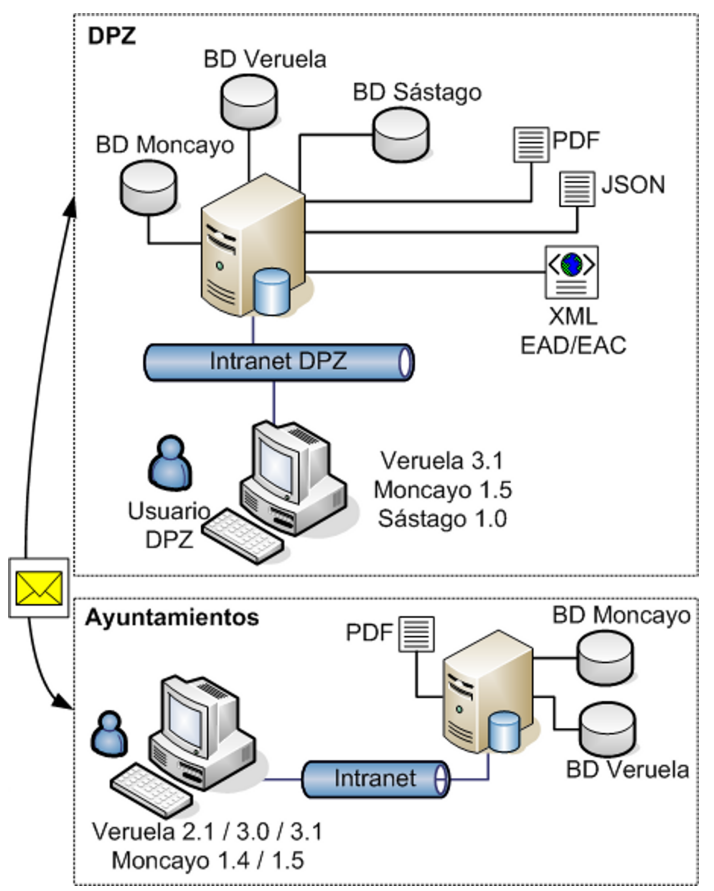

Figura 1. Esquema global de trabajo con las herramientas informáticas de gestión de archivos municipales, en la DPZ y los ayuntamientos

Las BBDD relacionales de Moncayo y Veruela mantienen gran parte de la información necesaria para el cumplimiento de la norma $\operatorname{ISAD}(\mathrm{G})$, aunque su diseño no contempló la adaptación a dicha norma. La aplicación Sástago, en cambio, nace para la gestión de descripción archivística normalizada, y para la exportación de contenidos. Su BD está diseñada internamente según las normas ISAD(G), ISAAR, ISDF e ISDIAH.

Sástago recoge de la BD de Veruela, por un lado, los contenidos necesarios para transformarlos de acuerdo con la norma $\operatorname{ISAD}(G)$; y por otro, parte de la información necesaria para la norma ISAAR. El resto de la información de estas normas, junto con los contenidos de las normas ISDF e ISDIAH se cumplimentan de forma manual.

Cada una de las aplicaciones gestiona su propia base de datos y las tres aplicaciones son complementarias. Están relacionadas a través de la base de datos de Veruela, a la que acceden con diferentes propósitos. En la figura 2 se representa cómo Moncayo exporta automáticamente las fichas a la BD de Veruela; y Sástago importa contenidos de las fichas para cargar parte de su propia base de datos. 


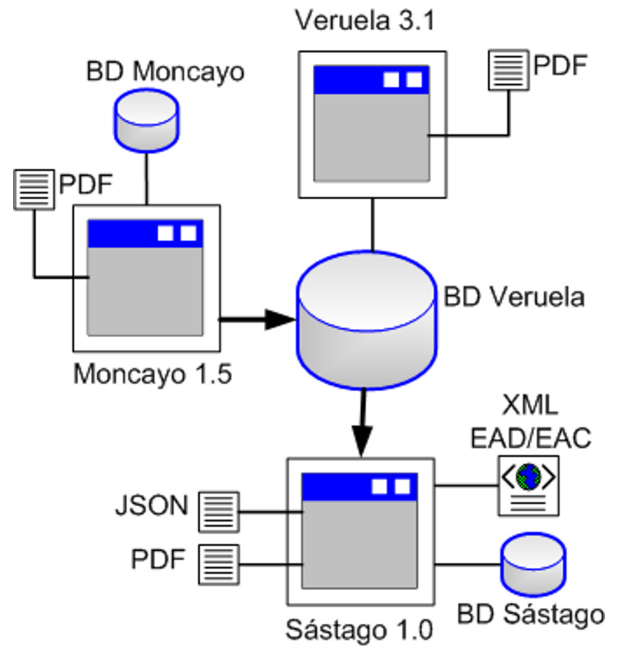

Figura 2. Relaciones entre las aplicaciones

El flujo de trabajo con estas aplicaciones es el siguiente: Los usuarios de Moncayo crean, describen y mantienen todos los documentos hasta el fin de su tramitación. Posteriormente se remite a la BD de Veruela de forma automática, transfiriendo de la BD de Moncayo los elementos de descripción necesarios y ficheros adjuntos que hubieran sido creados en el expediente. Veruela recibe automáticamente los datos de Moncayo, y además permite introducir de forma manual las fichas descriptivas de la documentación que no ha sido gestionada con Moncayo. Finalmente, para disponer de los contenidos de los fondos documentales en otros formatos se ha desarrollado Sástago. Todas las aplicaciones permiten la generación personalizada de informes en formato PDF.

\section{Moncayo}

Moncayo es un programa de gestión del archivo de oficina, pensado para los ayuntamientos de los municipios que -en los parámetros demográficos de nuestra provincia- podemos considerar como medianos y grandes, a partir de unos 3.000 habitantes, y que disponen de un número de empleados trabajando en sus oficinas relativamente alto.

Entendemos que Moncayo es especialmente útil para llevar un seguimiento del estado en que están en cada momento los documentos más habituales en nuestra administración: los expedientes, desde que se originan hasta que, una vez finalizados, se remiten al archivo central; lo cual no significa que esta aplicación informática no resulte adecuada también para otra clase de documentos. Hemos de indicar que los municipios en los que trabajamos únicamente tienen un archivo de oficina y otro para todas las de- más fases de los documentos, al que llamaremos central para simplificar.

Con Moncayo pretendemos: 1) que las personas encargadas de la tramitación de los documentos los tengan controlados en todo momento; 2) también que - para ello- vayan haciendo unas descripciones de los mismos siguiendo los criterios archivísticos facilitados por los técnicos de la Diputación zaragozana; y 3) que clasifiquen la documentación según un cuadro de clasificación de fondos orgánico-funcional, elaborado también por los archiveros de la institución provincial.

Con ello queremos lograr la existencia de unas descripciones y de unas clasificaciones suficientemente normalizadas, que resulten familiares al personal encargado de la tramitación de los documentos, lo cual facilitará su recuperación tanto con Moncayo como, ya en el archivo central, con Veruela, pues se puede pasar la información de aquél a éste. Conviene resaltar la importancia de que el personal municipal sepa cómo encontrar la documentación, pues los principales usuarios de sus archivos son los propios ayuntamientos.

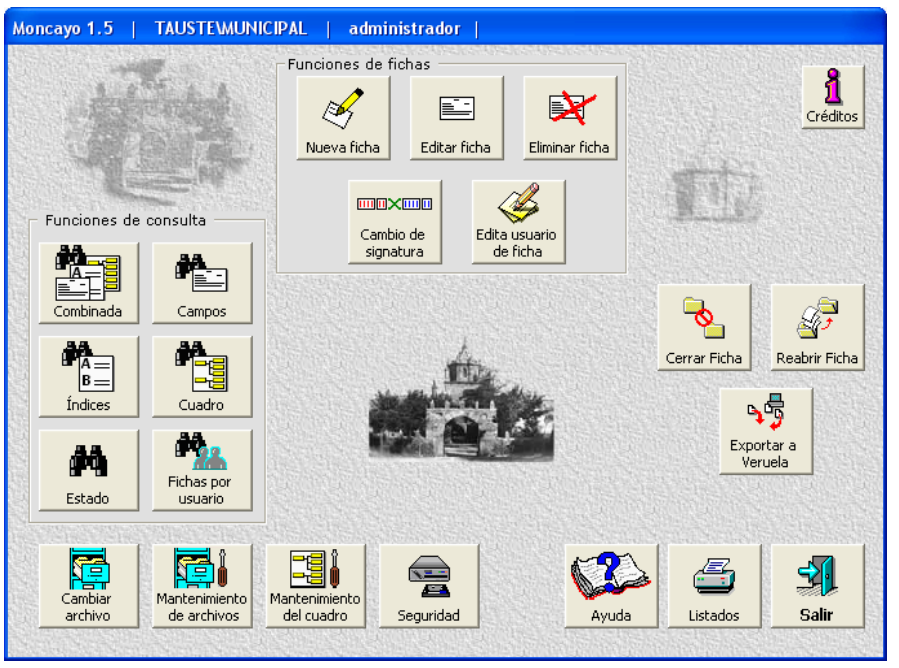

Figura 3. Moncayo 1.5: Menú principal

El flujo de trabajo para la creación de un documento se inicia a partir de la pantalla inicial (figura 3) seleccionando la opción nueva ficha, que nos lleva a cumplimentar el campo signatura. Así pues, para tener controlado físicamente el documento desde su inicio, lo primero que debemos decirle al ordenador es dónde (generalmente en qué caja y qué número hace dentro de la caja) va a estar depositado.

Después nos invita a describir el documento mediante un formulario (figuras 4 y 5 ) con varios campos, que se cumplimentan con estos datos: 
la fecha de inicio (con el formato día-mes-año); el productor, identificado en el cuadro de clasificación; la descripción, donde se hace una regesta del documento; notas, para aquella información que se considere necesaria y no corresponda su cumplimentación a ningún otro campo (no es obligatorio rellenar este campo); lugar, que por defecto aparece cumplimentado con el nombre del municipio, por ser, lógicamente, donde se van a producir la mayoría de documentos que gestiona, pero que puede cambiarse en caso necesario; estado, que es activo mientras se tramita el documento; tamaño, que por defecto es folio, el más común en nuestra administración, pudiéndolo cambiar en caso necesario; y soporte, que por defecto nos indica papel, pero que puede modificarse si es otro diferente.

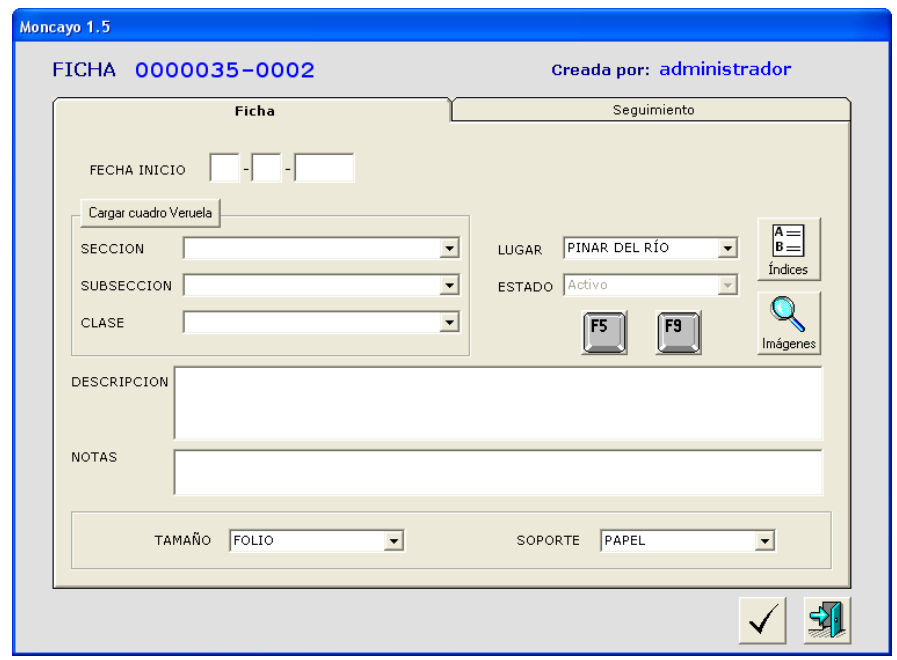

Figura 4. Moncayo 1.5: Nueva ficha

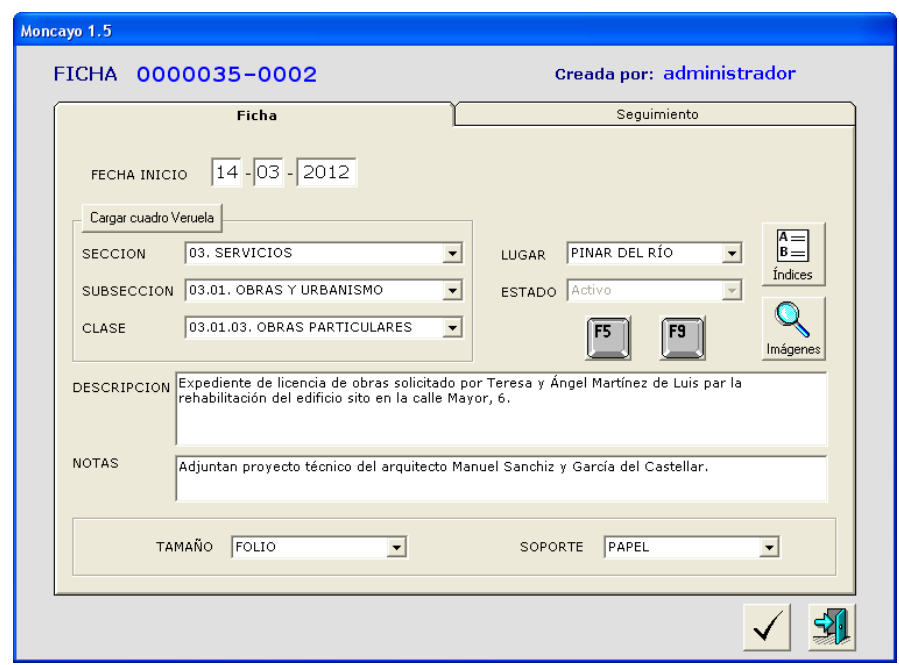

Figura 5. Moncayo 1.5: Ficha cumplimentada

A partir de esta fase de cumplimentación se pueden añadir ficheros informáticos en cualquier formato, por ejemplo, imágenes del propio documento que describimos, de forma que podrá ser consultado desde los ordenadores en red por diferentes usuarios, sin necesidad de manipular el original en papel.A continuación se pueden incluir los índices a los que esta ficha haya dado lugar, en estos cinco tipos: onomásticos, topónimos, organismos, cargos y profesiones (figura 6).

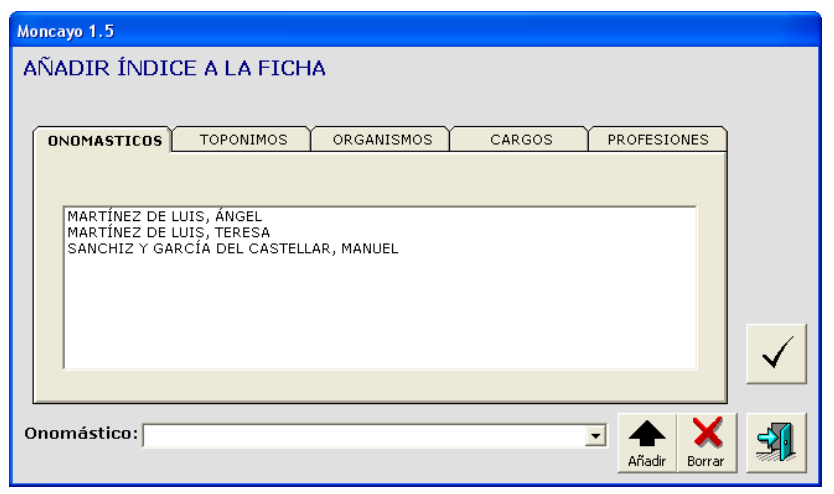

Figura 6. Moncayo 1.5: Índices

Cumplimentada la ficha inicial que identifica al documento, en caso de ser éste compuesto podemos ir describiendo los diferentes documentos simples que lo componen conforme se van generando, pulsando la opción seguimiento. En esta misma pantalla está el campo anotaciones, con el listado de los documentos simples que se han ido describiendo.

La recuperación de la información se puede realizar desde la pantalla inicial, pulsando el grupo de opciones funciones de consulta (figura 3 ), a partir de cualquiera de los campos cumplimentados en la pantalla ficha, por cualquiera de los índices introducidos, por el estado de tramitación, por el usuario, o por una combinación de dichas opciones.

Cuando el documento pase al archivo central, la descripción realizada en Moncayo se exporta a Veruela (figura 7 , en la página siguiente).

Otra de las necesidades detectadas en archivos de los municipios donde trabajamos es la necesidad de llevar un control de los usuarios que sea eficaz y eficiente. Para ello, en Moncayo 1.5 disponemos de una opción que registra los datos fundamentales de las consultas y préstamos de documentación, permitiendo imprimir las correspondientes hojas para que sean firmadas por los usuarios. Esta opción queda sobradamente justificada por muchos motivos: saber dónde está la documentación que ha salido del archivo; conocer cuál es la más solicitada, a efectos de posibles políticas de digitalización, de 
difusión, etc.; incluir en las memorias del archivo los datos estadísticos sobre los documentos servidos; etc.

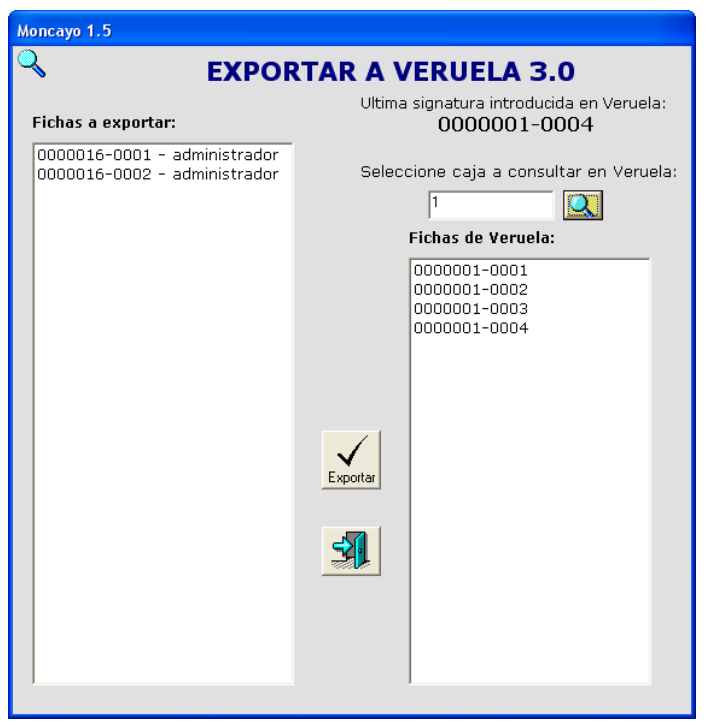

Figura 7. Moncayo 1.5: Exportación

\section{Veruela}

Veruela 3.1 es un programa concebido para la gestión interna del archivo, y de sus modificaciones con relación a la versión anterior interesan destacar las que tienen como objetivo adecuarlo a las normas vigentes. En concreto, con relación a los elementos esenciales establecidos en $\operatorname{ISAD}(G)$, se han realizado las siguientes modificaciones, que afectan al área de identificación: en cada registro descriptivo de Veruela3 se recoge de forma automática el código de identificación del fondo, elaborado desde la Diputación Provincial (que de este modo está supliendo la falta de una agencia oficial que asigne dichos códigos), siguiendo los criterios de la Norma Española de Descripción Archivística (NEDA). Dicho código se completa en cada registro con los del nivel de descripción que le corresponda. Respecto al título, se puede indicar si éste es el formal o si es atribuido. Las fechas se adecúan fundamentalmente a los criterios de la NEDA. Los restantes elementos considerados esenciales por las $\operatorname{ISAD}(G)$ no han precisado de cambios con relación a las versiones anteriores de Veruela.

Otro cambio importante en Veruela 3.1 con relación a la versión anterior afecta al control de usuarios y la gestión de préstamos, que se realizan igual que en Moncayo 1.5. El flujo de trabajo para la creación de un documento se inicia con la opción de crear ficha, donde se describe el documento con su regesta, las datas y su productor (figura 8). A continuación se pueden incluir los índices a los que esta ficha haya dado lugar, en sus diferentes tipos: onomásticos, topónimos, organismos, cargos y profesiones (figura 9).

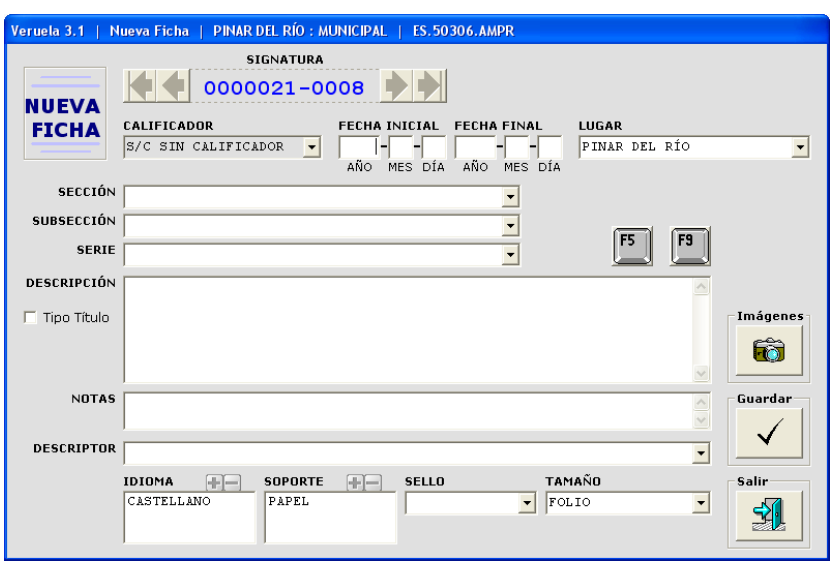

Figura 8. Veruela 3.1: Nueva ficha

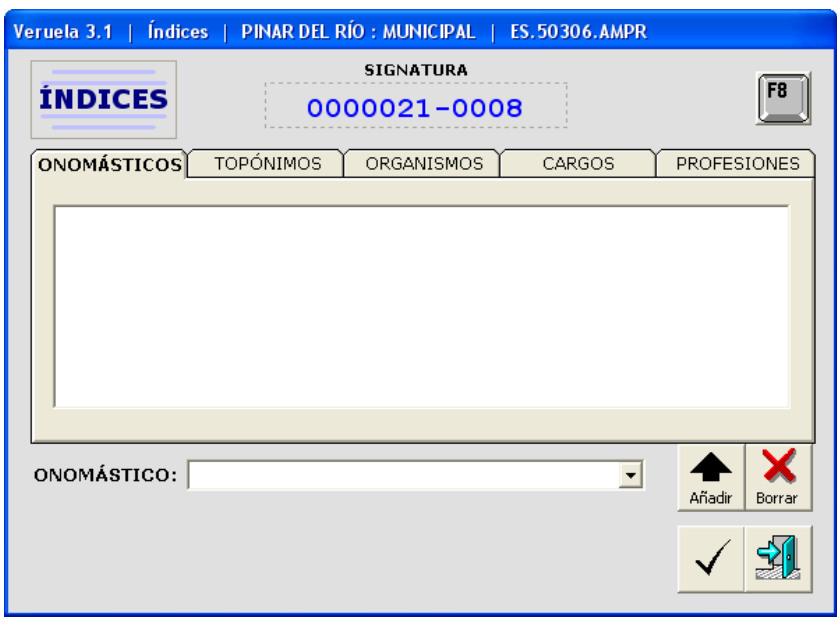

Figura 9. Veruela 3.1: Índices

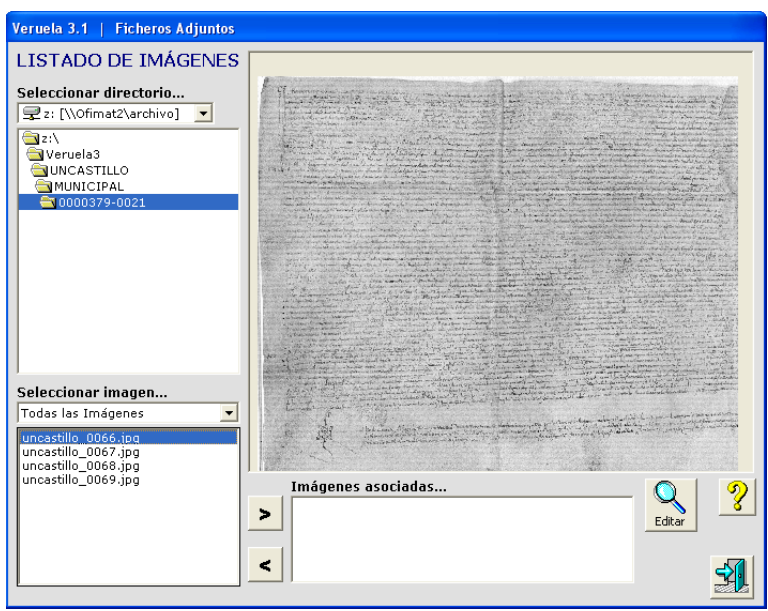

Figura 10. Veruela 3.1: Archivos adjuntos para la gestión de archivos en el ámbito municipal de la provincia de Zaragoza. // Ibersid. 6 (2012) 45-52. ISSN 1888-0967. 
También Veruela ofrece la posibilidad de adjuntar ficheros en cualquier formato. De esta forma se complementa la descripción con las partes del propio documento que se haya digitalizado, imágenes, contenidos multimedia, etc. (figura 10 , en la página anterior). La recuperación de la información se realiza de forma muy parecida a Moncayo.

\section{Sástago}

Sástago, por su parte, es un programa concebido para gestionar los contenidos de $\operatorname{ISAD}(G)$, ISAAR, ISDF e ISDIAH, y para generar archivos de intercambio de datos normalizados.

Sástago se crea con dos objetivos principales: difundir las descripciones documentales y recuperar los contenidos de las bases de datos, para una posible inclusión en otros sistemas informáticos. Con este fin, se permite la exportación de los contenidos de las normas en diferentes formatos: EAD, EAC, JSON y PDF. Las implementaciones para la exportación de contenidos de ISDF e ISDIAH se desarrollarán cuando se publiquen los formatos correspondientes.

De este modo es factible la interoperabilidad técnica mediante la funcionalidad ofrecida en Sástago para importar y exportar contenidos de descripción en formatos normalizados.

Estos ficheros podrán ser utilizados para difundir, a través de internet, el patrimonio documental de los archivos municipales.

El flujo de trabajo en esta aplicación es diferente según la norma. La principal diferencia está en que las normas ISAD(G) e ISAAR pueden importar datos de Veruela para algunos campos de sus áreas. Las normas ISDF e ISDIAH deben cumplimentarse de forma manual. Es común a todas ellas la edición de contenidos para su cumplimentación o modificación.

Con la ISAD(G) trabajando a nivel de fondo, se cargan de forma automática los siguientes campos: en el área de identificación, el código de referencia, título, fecha(s), nivel de descripción, y volumen y soporte (figura 11). En el área de contexto, el nombre del productor del fondo, y en el área de contenido y estructura, la organización.

Con ISAAR se importan los índices onomásticos y de instituciones, cumplimentando automáticamente el campo forma autorizada del nombre del área de identificación (figura 12).

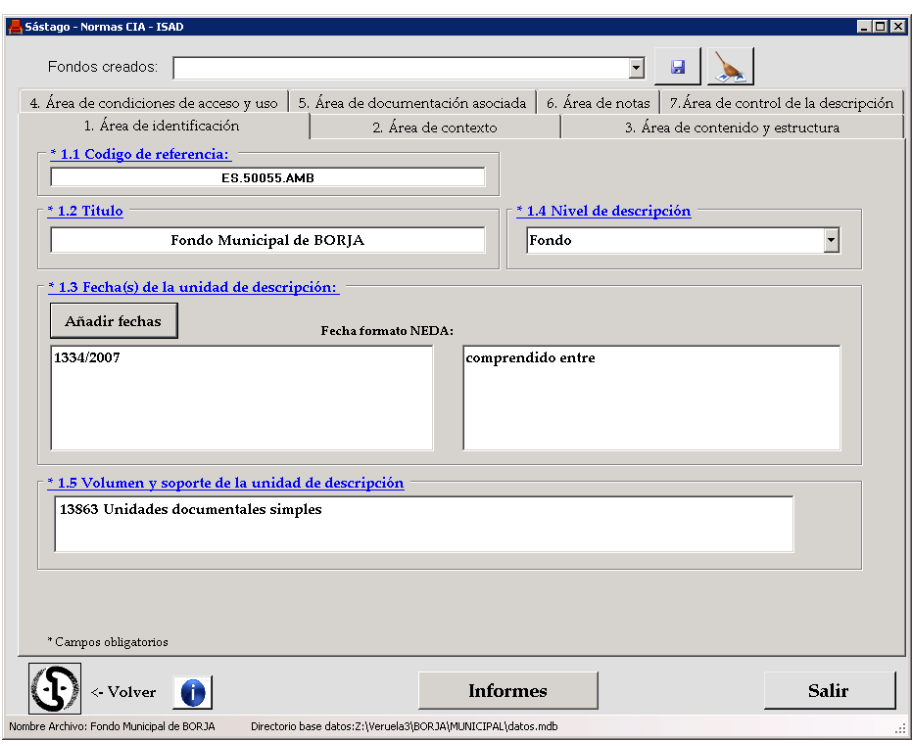

Figura 11. Sástago: carga automática ISAD(G) de los datos de Veruela

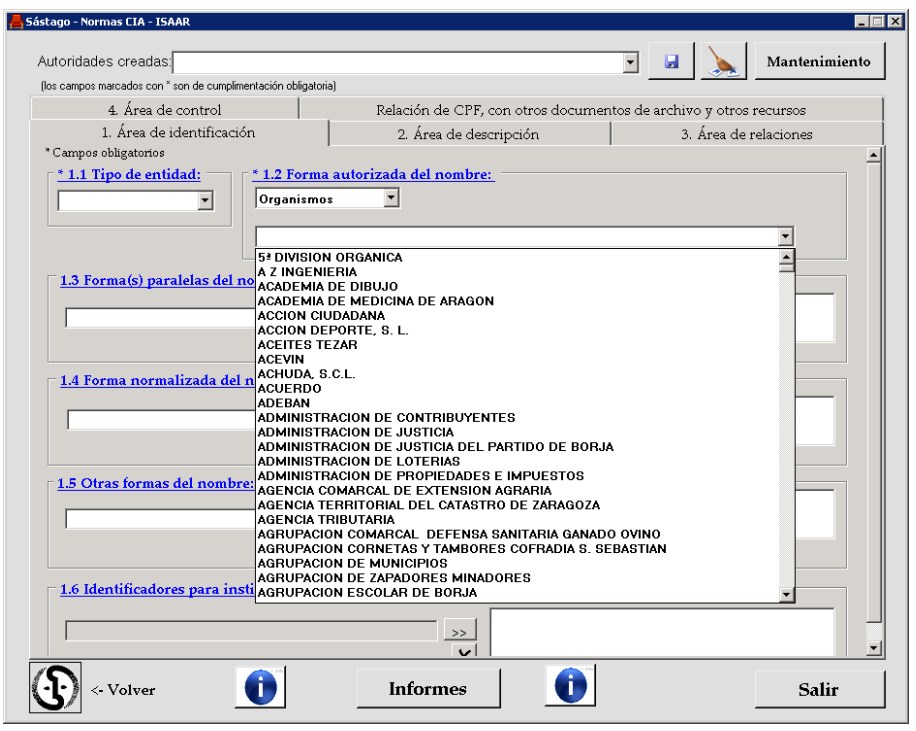

Figura 12. Sástago: carga automática de autoridades de ISAAR a partir de índices de Veruela

La creación de los ficheros de salida para $\operatorname{ISAD}(G)$ e ISAAR se inicia con la carga automática de datos. A continuación se cumplimentarán manualmente los campos vacíos de los que se conoce el contenido. $Y$ para finalizar se selecciona el formato deseado de exportación (figura 13), que puede ser EAD/EAC (figuras 14 y 15), JSON o PDF. 


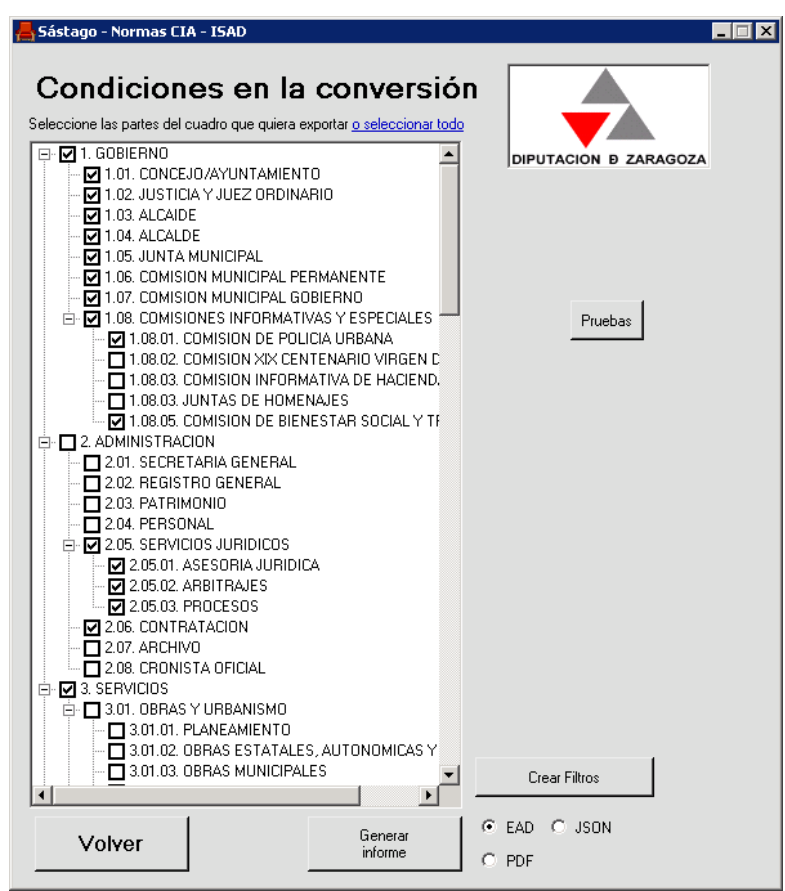

Figura 13. Sástago: exportación de datos

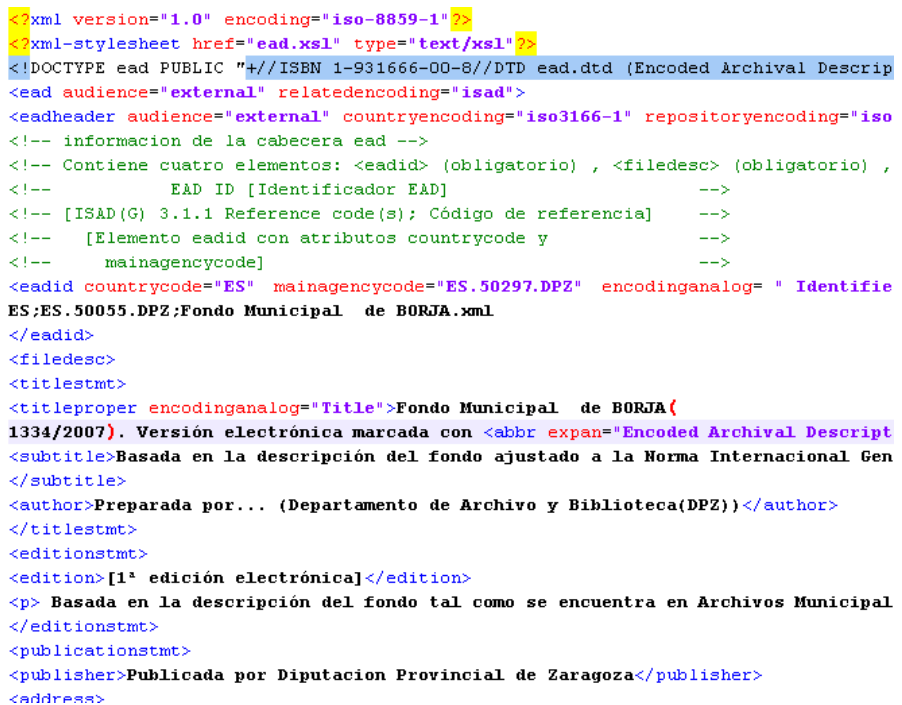

Figura 14. Sástago: fragmento de archivo EAD generado

Los contenidos de la $\operatorname{ISAD}(\mathrm{G})$ sólo permanecen en la BD de Sástago de forma temporal, durante el proceso de creación de los ficheros EAD de exportación. De esta forma, los contenidos del EAD siempre se recogen de Veruela, que contiene la información actualizada.

El resto de las normas ISAAR, ISDF e ISDIAH, por su propia naturaleza, son más estables en sus contenidos, por lo que permanecen en la base de datos hasta que el usuario los elimine.

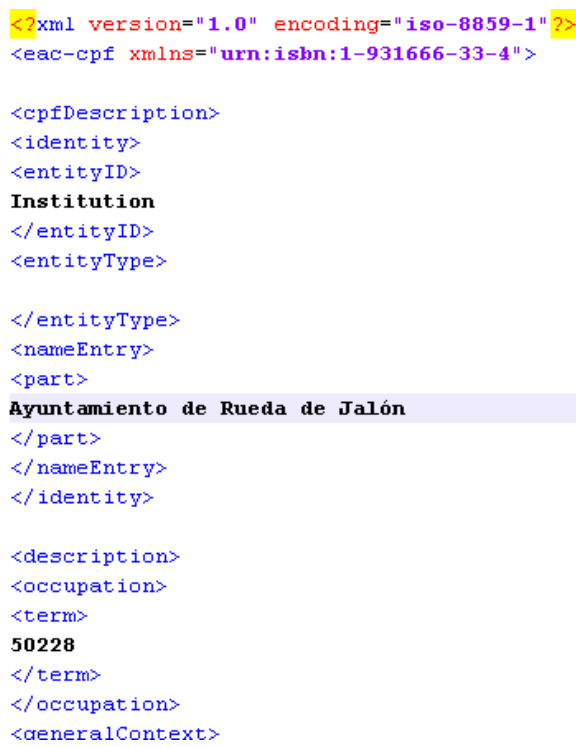

Figura 15. Sástago: fragmento de archivo EAC generado

\section{Conclusiones}

La gran mayoría de ayuntamientos de la provincia de Zaragoza necesitan la ayuda de otras instituciones para disponer de un servicio de archivos acorde con las actuales demandas sociales.

Con este conjunto de herramientas informáticas se cubren las necesidades de gestión del archivo de los municipios de las características de los aragoneses.

Se avanza considerablemente en la difusión normalizada del patrimonio archivístico al permitir la exportación de contenidos EAD, EAC, JSON y PDF. También se asegura la recuperación de contenidos de las descripciones documentales gestionadas.

Los documentos EAD y EAC generados por Sástago pueden ser importados por la herramienta de difusión ICA-AtoM, de software libre, ofrecida por el CIA.

\section{Notas}

(1) Sobre la labor realizada por el Departamento de Archivos Municipales de la Diputación Provincial de Zaragoza, vid. Diputación Provincial de Zaragoza (2009). Sobre la forma de trabajar con los ayuntamientos, vid. Ferrer y Egea (2008).

(2) Sobre el programa informático utilizado en los primeros años, vid. Egea et alii (1996).

\section{Referencias}

Consejo Internacional de Archivos (2000). ISAD(G).Norma internacional general de descripción archivística. $2^{\mathrm{a}}$ ed. Madrid: Subdirección General de los Archivos Estataleshttp://www.mcu.es/archivos/docs/isad.pdf (2012-04-09). para la gestión de archivos en el ámbito municipal de la provincia de Zaragoza. // Ibersid. 6 (2012) 45-52. ISSN 1888-0967. 
Consejo Internacional de Archivos (2004). ISAAR (CPF). Norma Internacional sobre los registros de autoridad de archivos relativos a instituciones, personas y familias, 2 . $^{a}$ ed., 2004. http://www.mcu.es/archivos/docs/isaar.pdf. (2012-04-09).

Consejo Internacional de Archivos (2007). ISDF.Norma internacional para la descripción de funciones. $1^{\mathrm{a}}$ ed. París: CIA, 2007. http://www.mcu.es/archivos/docs/CE/ ISDF_ESP_definitiva.pdf (2012-04-09).

Consejo Internacional de Archivos (2004). ISDIAH. Norma Internacional para describir instituciones que custodian fondos de archivo. París, 2008. http://www.mcu.es/ archivos/docs/CE/ISDIAH_ESP.pdf (2012-04-09).

Diputación Provincial de Zaragoza (2009). La DPZ y los Archivos Municipales. (2009). http://cultura.dpz.es/es/ archivos-y-bibliotecas/archivos-de-la-provincia/archivosmunicipales/la-dpz-y-los-archivos-municipales/id/66 (2012-04-09).

Egea Gilaberte, José Francisco; et alii (1996). El programa informático de la Diputación de Zaragoza para los archivos municipales y su aplicación en los de los juzgados municipales de la provincia. // Actas de las VI jornadas de archivos aragoneses: Situación y perspectivas de los archivos de la administración de justicia. Zaragoza: Diputación General de Aragón, Departamento de Educación y Cultura, 1996. 221-236.

Ferrer, Blanca y Egea, José F. (2008): La gestión compartida: hacia la creación del subsistema de archivos municipales de la Diputación de Zaragoza. // Compartir archivos: Actas de las VIII Jornadas de Archivos Aragoneses. Huesca: Gobierno de Aragón; Diputación Provincial, 2008. Tomo I. Sistemas para compartir. 2008. 233-244. http://www.aragon.es/estaticos/GobiernoAragon/Departa mentos/EducacionCulturaDeporte/Documentos/docs/Are as/Jornadas/VIII jorndas aragonesas/12\%20La\%20Ges ti\%C3\%B3n\%20Compartida.pdf (2012-04-09).

Ministerio de Cultura. Norma española de descripción archivística (NEDA). $1^{\text {a }}$ versión. http://www.aefp.org.es/NS/ Documentos/NormasDescriptivas/NEDA/NEDA_v1_200 5_completa.pdf (2012-04-09).

Enviado: 2012-04-17. Versión corregida: 2012-09-13. Aceptado: 2012-09-13. 